\title{
The molecular mass function of the local Universe
}

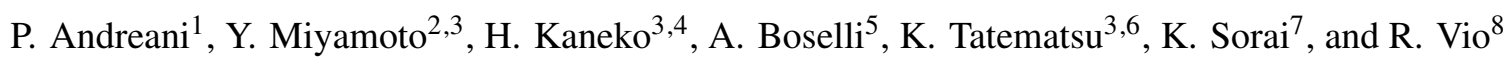 \\ 1 European Southern Observatory, Karl-Schwarzschild-Straße 2, 85748 Garching, Germany \\ e-mail: pandrean@eso.org \\ 2 National Astronomical Observatory of Japan, National Institutes of Natural Sciences, 2-21-1 Osawa, Mitaka, Tokyo 181-8588, \\ Japan \\ 3 Nobeyama Radio Observatory, National Astronomical Observatory of Japan, National Institutes of Natural Sciences, \\ 462-2 Nobeyama, Minamimaki, Minamisaku, Nagano 384-1305, Japan \\ 4 Joetsu University of Education, Yamayashiki-machi, Joetsu, Niigata 943-8512, Japan \\ 5 Aix Marseille Univ., CNRS, CNES, LAM, Marseille, France \\ 6 Department of Astronomical Science, SOKENDAI (The Graduate University for Advanced Studies), 2-21-1 Osawa, Mitaka, Tokyo \\ 181-8588, Japan \\ 7 Department of Physics, Faculty of Science, Hokkaido University, Kita 10, Nishi 8, Kita-Ku, Sapporo, Hokkaido 060-0810, Japan \\ 8 Chip Computers Consulting s.r.l., Viale Don L. Sturzo 82, S. Liberale di Marcon, 30020 Venice, Italy
}

Received 16 June 2020 / Accepted 6 October 2020

\section{ABSTRACT}

\begin{abstract}
Aims. We construct the molecular mass function using the bivariate $K$-band-mass function (BMF) of the Herschel Reference Survey (HRS), which is a volume-limited sample that has already been widely studied at the entire electromagnetic spectrum.

Methods. The molecular mass function was derived from the $K$-band and the gas mass cumulative distribution using a copula method, which is described in detail in our previous papers.

Results. The $\mathrm{H}_{2}$ mass is relatively strongly correlated with the $K$-band luminosity because of the tight relation between the stellar mass and the molecular gas mass within the sample with a scatter, which is likely due to those galaxies which have lost their molecular content because of environmental effects or because of a larger gas consumption due to past star formation processes. The derived $\mathrm{H}_{2}$ MF samples the molecular mass range from $\sim 4 \times 10^{6} M_{\odot}$ to $\sim 10^{10} M_{\odot}$, and when compared with theoretical models, it agrees well with the theoretical predictions at the lower end of the mass values; whereas at masses larger than $10^{10} M_{\odot}$, the HRS sample may miss galaxies with a large content of molecular hydrogen and the outcomes are not conclusive. The value of the local density of the molecular gas mass inferred from our analysis is $\sim 1.5 \times 10^{7} M_{\odot} \mathrm{Mpc}^{-3}$, and it is compared with the results at larger redshifts, confirming the lack of strong evolution for the molecular mass density between $z=0$ and $z=4$.

Conclusions. This is the first molecular mass function that has been derived on a complete sample in the local Universe, which can be used as a reliable calibration at redshift $z=0$ for models aiming to predict the evolution of the molecular mass density.
\end{abstract}

Key words. ISM: molecules - submillimeter: galaxies - galaxies: statistics - galaxies: luminosity function, mass function

\section{Introduction}

Understanding galaxy formation and evolution is still at its infancy and the complete picture that fully describes how galaxies form and evolve into structures has not been fully outlined yet. One way to address this issue is through the careful comparison between the predictions of theories (and simulations) with the observations. The classical approach is the use of the statistical nature of the galaxy properties and the determination of it over various sets of galaxy populations (i.e. at different redshifts, types, environment, etc.).

In many branches of astronomy and cosmology, a very common tool used to investigate the statistical properties of a population of objects is the luminosity and the mass function (e.g. Johnston 2011). In the study of the physical processes that govern galaxy formation and evolution, the stellar and gas mass functions (MFs) in particular constitute one of the fundamental pillars to constrain theories from observations.

Observationally, galaxy evolution can be followed by studying the baryons (stars and gas) which are supposed to be associated with the dark matter (DM) haloes. The gas cools and condenses within the haloes, until it produces an independent self-gravitating unit which can form stars, which heats and enriches the rest of the gas and perhaps even ejects it from the halo (Mo et al. 1998).

In this framework, hydrodynamical simulations as well as semianalytical and empirical approaches add the baryonic component to the DM in haloes and follow their evolution through a merger history including gas and star formation processes. These star formation and feedback processes are not understood in detail (see for reviews, Mo et al. 2010; Somerville \& Dave 2015).

To tune these multi-parameter models, their outcomes are compared to the statistical properties of galaxies in the local Universe. As mentioned above, key constrains are the mass and luminosity functions (LFs; i.e. Dave et al. 2017; Lagos et al. 2016).

From the observational side, in order to compute these statistics, it is necessary to build large samples of galaxies that are selected at various wavelengths. The derived LFs and MFs contain some significant uncertainties mainly due to the lack of either the imaging of large fields or the required multi-wavelength homogeneous coverage and complete redshift information. 
Molecular gas and star formation rate. Observations widely point out that star formation (SF) occurs within dusty molecular clouds (i.e. for reviews Solomon \& van den Bout 2005; Kennicutt \& Evans 2012) and that the surface density of the star formation rate (SFR) correlates with the surface density of molecular hydrogen, $\mathrm{H}_{2}$, which roughly follows a linear relation, $\sigma(\mathrm{SFR}) \propto \sigma\left(\mathrm{H}_{2}\right)$ (e.g. Bigiel et al. 2008; Schruba et al. 2011).

The $\mathrm{H}_{2}$ content of galaxies has been studied most through the emission from ${ }^{12} \mathrm{CO}$ as a tracer. Measuring $\mathrm{H}_{2}$ mass requires the definition of the $\mathrm{CO}-\mathrm{H}_{2}$ conversion factor, $\mathrm{X}_{\mathrm{CO}}$, in order to convert it from its observable tracer, $\mathrm{CO}$, which in and of itself is a function of metallicity (i.e. Bolatto et al. 2013) and stellar mass (i.e. Dessauges et al. 2017).

Reasons for a local molecular mass function. Previous molecular MFs of nearby galaxies have been indirectly derived from the $\mathrm{CO}$ luminosity distribution, which has also been built on assumptions of its relation with other tracers Keres et al. (2003) used an incomplete CO sample based on a far-infrared (FIR) selection and used the correlation of the $\mathrm{CO}$ with the $60 \mu \mathrm{m}$ luminosity. The resulting CO MF is, therefore, biased towards gas-rich galaxies. An updated estimate of the $\mathrm{H}_{2}$ mass function (hereafter $\mathrm{H}_{2} \mathrm{MF}$ ), based on an empirical and variable $\mathrm{CO}-\mathrm{H}_{2}$ conversion factor, $\mathrm{X}_{\mathrm{CO}}$, was presented by Obreschkow \& Rawlings (2009). Saintonge et al. (2017) and Fletcher et al. (2020) derived an $L^{\prime}(\mathrm{CO})$ luminosity distribution from the CO legacy data base for the GASS survey (COLD GASS, Saintonge et al. 2011). This survey, although biased towards massive galaxies (stellar mass, $M_{\star}>$ a few $10^{9} M_{\odot}$, Saintonge et al. 2011, 2017), that is to say it might not sample a sufficiently large dynamic range in $M_{\star}$ to trace a fair distribution, is at present the only survey with a large enough database to allow for a fair reconstruction of the $L^{\prime}(\mathrm{CO})$ luminosity distribution. However, this sample is also biased because it is not blindly selected and its selection from the SSDS survey is prone to many uncertainties due to unknown and uncontrolled systematics.

The Herschel ${ }^{1}$ Reference Survey (HRS) is a Herschel guaranteed time key project, performing photometric observations with the SPIRE cameras towards HRS galaxies (Boselli et al. 2010).

The survey selection criteria (magnitude- and volumelimited, see Sect. 2), size and multiwavelength coverage (from UV to radio wavelengths both in spectroscopy and photometry), together with the Herschel results in the FIR, which are sensitive to dust mass down to $10^{4} M_{\odot}$, have shown that the HRS can be considered as a 'reference' sample to carry out a statistical analysis in the local Universe (Boselli et al. 2010; Andreani et al. 2014, 2018).

In this Letter, we compute and discuss the local molecular mass function derived from the HRS sample. We note that $\mathrm{CO}$ observations are taken with single dishes and described in detail in other papers (Boselli et al. 2014a,b; Andreani et al., in prep.). Our aim is to infer the local molecular mass density on a more solid ground, which could be used as a reference to set limits on current models predicting the evolution of the molecular mass with redshift.

The paper is organised as follows. The sample with the additional observations that were carried out with the FOREST array at the Nobeyama $45 \mathrm{~m}$ antenna is briefly described in Sect. 2. The computation of the molecular mass function is found in Sect. 3 and discussed in Sect. 4.

\footnotetext{
1 Herschel is an ESA space observatory with science instruments provided by European-led Principal Investigator consortia and with important participation from NASA
}

\section{The data}

The HRS is a volume-limited sample (i.e. $15<D<25 \mathrm{Mpc}$ ), including late-type galaxies (LTGs; Sa and later) with a $2 \mathrm{MASS}$ $K$-band magnitude $\leq 12$ mag and early-type galaxies (ETGs; S0a and earlier) with $\leq 8.7 \mathrm{mag}$. Additional selection criteria are high Galactic latitude $\left(b>+55^{\circ}\right)$ and low Galactic extinction (AB $<0.2$ mag, Schlegel et al. 1998). The sample includes 322 galaxies (260 LTGs and 62 ETGs), and the total volume over an area of 3649 sq. deg. is $4539 \mathrm{Mpc}^{3}$. The selection criteria are fully described in Boselli et al. (2010).

This data set has been extensively used to investigate many aspects of the physics of the interstellar medium, among others the stellar, dust, gas mass functions, infrared luminosity function, and the star formation function (Cortese et al. 2012; Boselli et al. 2010, 2014a,b, 2015; Ciesla et al. 2014; Andreani et al. 2014, 2018).

We have recently investigated the HRS LFs and MFs using the bivariate functions with respect to the $K$-band luminosity, which is the band at which the sample is complete (Andreani et al. 2014, 2018). We have employed an accurate statistical method which makes use of the entire data sets including the upper limits. We have shown that the HRS samples galaxies down to a few $10^{8} M_{\star}$ and thus has a dynamic range that is sufficiently wide for an accurate determination of the $\mathrm{H}_{2}$ mass function.

We were able to construct the $\mathrm{H}_{2}$ MF of the HRS sample from the bivariate $K$-band luminosity- $\mathrm{H}_{2}$ mass function because of the good correlation (correlation coefficient, $\rho=0.67$ ) between the $K$-band luminosity and the $\mathrm{H}_{2}$ mass. Based on this finding, we could reconstruct the sample from the bivariate function and the analytical form of the $\mathrm{H}_{2}$ mass function (see for detail Andreani et al. 2014, 2018). Despite all the caveats described in the paper (Andreani et al. 2018), the molecular mass function is the first function to have been built on a complete sample, although the completeness is in the $K$-band.

In this work, we improve our analysis from Andreani et al. (2018) by making use of additional observations of the $\mathrm{CO}(1-$ 0 ) fluxes towards those galaxies previously lacking this measurement. Of the 322 galaxies, 225 observations are reported in Boselli et al. (2014a,b), 43 more have been targeted in JanuaryFebruary 2018 and 2019 with the $45 \mathrm{~m}$ radio telescope of the Nobeyama Radio Observatory $(\mathrm{NRO})^{2}$. Observations will be fully described, analysed, and discussed in Andreani et al. (in prep.).

Here we briefly outlined how the observations were taken. We used the FOur-beam REceiver System (FOREST: Minamidani et al. 2016) and employed the OTF mapping mode. The observed area varied according to the optical size of the galaxies from $2^{\prime \prime} \times 2^{\prime \prime}$ to $4^{\prime \prime} \times 4^{\prime \prime}$. The total time for the observations varied between a couple of hours to $8 \mathrm{~h}$ depending on the expected CO line flux.

We used the $2 \times 2$ focal-plane dual-polarisation sidebandseparating SIS mixer receiver for the single side band (SSB) operation of FOREST, which provides eight intermediate frequency (IF) paths (i.e. four beams $\times$ two polarisations) independently. The backend was an FX-type correlator system, SAM45, which consists of 16 arrays with 4096 spectral channels each. The frequency coverage and the resolution for each array was set to $2 \mathrm{GHz}$ and $488.24 \mathrm{kHz}$, respectively, which gives a velocity coverage and resolution of $\sim 10000$ and $1.5 \mathrm{~km} \mathrm{~s}^{-1}$ at $115 \mathrm{GHz}$.

At $115 \mathrm{GHz}$, the half-power beam width of the $45 \mathrm{~m}$ telescope with FOREST is $\sim 14^{\prime \prime}$ and it degrades to $\sim 17^{\prime \prime}$ because of

\footnotetext{
2 Nobeyama Radio Observatory is a branch of the National Astronomical Observatory of Japan, National Institutes of Natural Sciences.
} 
a scanning effect. The system noise temperature is $300-500 \mathrm{~K}$ during the observing runs. In order to check the absolute pointing accuracy, we observed a pointing source (3C 273, rtvir, r-uma, rleo) using a $43 \mathrm{GHz}$ band receiver every hour.

The line intensity was calibrated by the chopper wheel method, yielding an antenna temperature, $T_{\mathrm{A}}^{\star}$, which was corrected for both atmospheric and antenna ohmic losses. The main beam brightness temperature, $T_{\mathrm{mb}}$ was converted from $T_{\mathrm{A}}^{\star}$ for each IF by observing a standard source, the carbon star IRC +10216 . The scaling factors not only correct the main-beam efficiency $\left(\eta_{\mathrm{mb}}\right)$ of the $45 \mathrm{~m}$ antenna, but they also compensate for the decrease in line intensity due to the incompleteness of the image rejection for the SSB receiver (e.g. Nakajima et al. 2013) (see Sorai et al. 2019, for more details).

The new observations refer to those objects with the lowest $K$-band luminosities and therefore allowed us to sample the lowest end of the molecular and stellar masses, down to values of the mass function of $\sim 4 \times 10^{6} M_{\odot}$. We made use of the total $268(225$ from Boselli et al. 2014a and 43 from Andreani et al., in prep.) galaxies of which we have 183 detections and 85 upper limits to obtain and discuss the $\mathrm{CO}$ distribution function and the molecular mass function derived from the $\mathrm{CO}(1-0)$ observations.

\section{The $C O(1-0)$ luminosity and the molecular mass}

The construction of a mass function is very sensitive to the quality of the sample selection. In order to have as much homogeneity as possible across different observations, we recomputed the $\mathrm{CO}(1-0)$ line luminosities for all galaxies following Boselli et al. (2014b).

In the HRS sample, we expect that because of the wide dynamic range in parameters (Boselli et al. 2012), the conversion factor, $\mathrm{X}_{\mathrm{CO}}$, significantly changes from massive, metal-rich quiescent to dwarf, metal poor galaxies and a constant $X_{C O}$ value may underestimate the molecular content at stellar masses below $10^{10} M_{\odot}$. We then followed Boselli et al. (2014b) and computed the molecular mass and the molecular mass function twice, applying a constant and a luminosity dependent $\mathrm{X}_{\mathrm{CO}}$ conversion factor.

The constant conversion factor is $\mathrm{X}_{\mathrm{CO}}=2.3 \times 10^{20} \mathrm{~cm}^{-2} /$ $\left(\mathrm{K} \mathrm{km} \mathrm{s}^{-1}\right) \quad\left(\alpha_{\mathrm{CO}}=3.6 M_{\odot} /\left(\mathrm{K} \mathrm{km} \mathrm{s}^{-1} \mathrm{pc}^{2}\right)\right)$. The luminositydependent $\mathrm{X}_{\mathrm{CO}}$ is $\log \mathrm{X}_{\mathrm{CO}}=-0.38 \times \log L_{H}+24.23\left[\mathrm{~cm}^{-2} /\right.$ $\left(\mathrm{K} \mathrm{km} \mathrm{s}^{-1}\right)$ ], where $L_{H}$ are the $H$-band luminosities available from 2MASS for all the HRS galaxies (Skrutskie et al. 2006) (see for further detail Boselli et al. 2014b). Hereafter, we refer to those values as $M\left(\mathrm{H}_{2}\right) c$ and $M\left(\mathrm{H}_{2}\right) v$.

Following a procedure similar to that applied in previous papers (Andreani et al. 2014, 2018), we computed the bivariate function of the two values of the $\mathrm{H}_{2}$ mass and the $K$-band luminosity using a copula method (see Hofert et al. 2018). The difference here with respect to the previous computation is that instead of the Gaussianisation of the data and the use of the Gaussian copula, the best copula among a set of copula families was selected by means of a maximum-likelihood approach (Hofert et al. 2018). In the present case, the best result is given by a rotated Tawn type 2 copula $\left(180^{\circ}\right)$.

The bivariate functions of the molecular masses and the $K$-band luminosity are shown in Fig. 1; for both cases, the molecular mass was derived with a luminosity-dependent and with a constant conversion factor, respectively. Both functions show a well defined correlation between these two values, which reflects the strong relation between the stellar mass and the molecular gas mass of the HRS sample (Boselli et al. 2014b; Andreani et al. 2018).

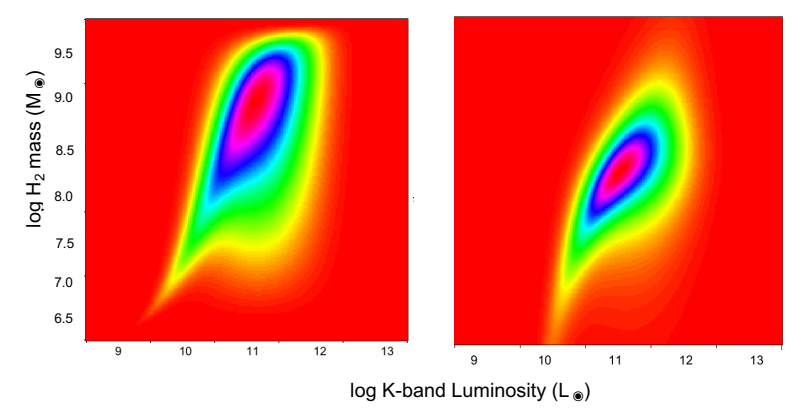

Fig. 1. Bivariate functions of the molecular masses and the $K$ band luminosity, with the molecular mass derived with a luminositydependent conversion factor (left) and a constant (right), respectively. The colour code refers to the correlation between the two variables (i.e. larger for those lying in the pink region). The spread is larger for the luminosity-dependent conversion factor. In this latter case, galaxies with a larger $K$-band luminosity have a lower molecular mass content, while those of a lower $K$-band luminosity have a large molecular mass content (see text).

The overall spread seen in Fig. 1 may be due to a slightly lower molecular mass content in HI-deficient galaxies (Boselli et al. 2014c; Andreani et al. 2018). However, the dynamic range and the overall spread are larger for the bivariate function derived with a luminosity-dependent conversion factor. In this latter case, we assigned a lower molecular content to those galaxies with a larger $K$-band luminosity and a larger molecular gas content to those with a lower $K$-band luminosity. The ratio between the molecular gas to the stellar mass, $M\left(\mathrm{H}_{2}\right) / M_{\star}$, decreases when the stellar mass increases, as can be seen in the scaling relation in Boselli et al. (2014b). This means that in the most massive galaxies, the gas was already transformed into stars; whereas in the dwarf galaxies, this process has not yet stopped and therefore the gas content is larger at lower stellar mass.

Figure 2 displays the $\mathrm{H}_{2}$ MF derived from the BMF for the two cases (constant and luminosity-dependent $\mathrm{X}_{\mathrm{CO}}$ in pink and red, respectively). The shown curves correspond to the Johnson families used to infer the bivariate function (see detail in Vio et al. 1994; Andreani et al. 2018). As widely explained in our previous papers (Andreani et al. 2018), these derived functions are the closest approximation to the analytical form of the corresponding mass functions. We could infer the values of these latter functions down to the lower end of the MFs, that is to molecular masses of $\sim 4 \times 10^{6} M_{\odot}$. The errorbars shown in Fig. 2 were derived by extracting the $\mathrm{H}_{2} \mathrm{MF}$ from the BMF computation allowing for the variation of the input variables within their observation errorbars. Table 1 reports the average values in every $M\left(\mathrm{H}_{2}\right)$ mass bin of the $\mathrm{H}_{2} \mathrm{MF}$ values of the functions extracted from the bivariate computation for the two $\mathrm{X}_{\mathrm{CO}}$ cases (constant and luminosity-dependent $\mathrm{X}_{\mathrm{CO}}$ ).

We compare the molecular mass functions of the HRS sample with that obtained by Fletcher et al. (2020) for the COLD GASS sample. This comparison must be considered with caution and it is only indicative. In addition to the issues discussed in the Introduction, we lack information about the galaxy properties needed to apply the luminosity dependent conversion factor between $L^{\prime}(\mathrm{CO})$ and $\mathrm{H}_{2}$ equal to the $\mathrm{X}_{\mathrm{CO}}$ factor used in this work (Boselli et al. 2014b). Therefore, the Fletcher et al. (2020)'s values can only be strictly compared to the curve obtained with the $M\left(\mathrm{H}_{2}\right) c$ values (pink curve in Fig. 2).

The new $\mathrm{CO}(1-0)$ measurements allow for one to sample the molecular mass function at a factor of ten deeper than that of 


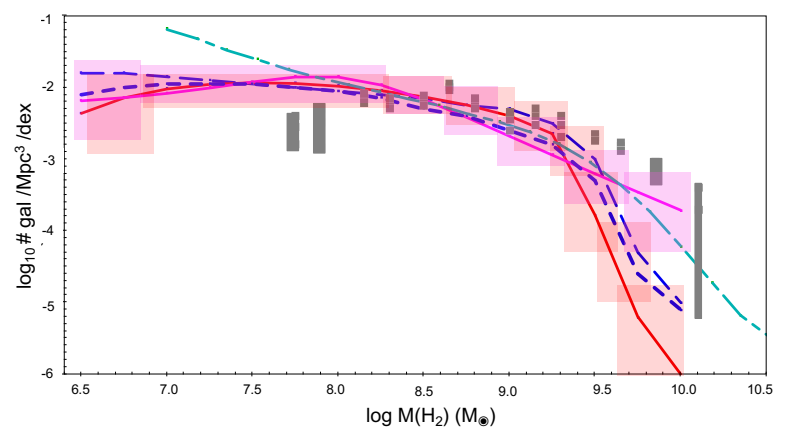

Fig. 2. Molecular mass functions derived in this work using the parameters of the bivariates' $K$-band luminosity- $\mathrm{H}_{2}$ mass. The red refers to the $\mathrm{H}_{2}$ MF derived for a luminosity dependent $\mathrm{X}_{\mathrm{CO}}$ factor, while the pink curve corresponds to that derived for a constant $\mathrm{X}_{\mathrm{CO}}$. The blue curves refer to the models reported in Lagos et al. (2015), while the green curve corresponds to that by Popping et al. (2014). The dark grey bars refer to the values of the $\mathrm{H}_{2}$ MF by Fletcher et al. (2020), taken from their fiducial model. The errorbar boxes have been slightly displaced along the $x$-axis to avoid confusion with overlapping colours.

COLD GASS. The discrepancy shown at large mass values can be attributed, on the one hand, to the $\mathrm{X}_{\mathrm{CO}}$ factor. But even in the case of constant $\mathrm{X}_{\mathrm{CO}}$, the HRS $\mathrm{H}_{2} \mathrm{MF}$ is lower than the values from Fletcher et al. (2020) at large masses. The HRS sample misses galaxies with large molecular masses because its volume is too small to contain a significant number of large mass galaxies (Boselli et al. 2014b). Indeed, the number of galaxies with $M\left(\mathrm{H}_{2}\right) \sim 10^{10} M_{\odot}$ is only 5 . The errorbars at $M\left(\mathrm{H}_{2}\right)>3 \times 10^{9} M_{\odot}$ are correspondingly large. Large errorbars are also assigned to the higher end of the MF by Fletcher et al. (2020) and a fair comparison is difficult to make. At low masses, the curve of Fletcher et al. (2020) shows the incompleteness that is already at $M\left(\mathrm{H}_{2}\right)<10^{8} M_{\odot}$.

In Fig. 2, we added the predictions of theoretical models, either hydro-dynamical simulations (Lagos et al. 2015) or semianalytical (Popping et al. 2014). It is clear that the HRS sample results put stringent constrains on the available models at the low mass end. While both models by Lagos et al. (2015) agree with the HRS measurements, the one by Popping et al. (2014) seems to predict many more low mass galaxies than seen in the local Universe. It is also important to note that there is a discrepancy between the predictions of the models by Popping et al. (2014) and Lagos et al. (2015) at $M\left(\mathrm{H}_{2}\right)>6 \times 10^{9} M_{\odot}$.

\section{Discussion}

The molecular mass function reported in Fig. 2 is the first function to have been built on a complete sample, exploiting a bivariate analysis of the molecular gas mass and the $K$-band luminosity. Since the latter mainly traces the star mass, the observed relation is likely dominated by galaxies in which there is still molecular hydrogen where stars are located. The scatter seen in Fig. 1 is very likely to be attributed to those galaxies where the molecular hydrogen is deficient, as is observed in objects that are strongly influenced by environmental effects and/or in massive galaxies where the star formation activity is low (Boselli et al. 2014c; Andreani et al. 2018).

The bivariate analysis allowed us to derive a $\mathrm{H}_{2} \mathrm{MF}$ of the sample. The MF was compared with the predictions of semi-analytical models (Lagos et al. 2015; Popping et al. 2014) and shows good agreement with the models from Lagos et al. (2015) and a large discrepancy with that of Popping et al. (2014).
Table 1. $\mathrm{H}_{2}$ MF resulting from the bivariate and for the two chosen values of the $\mathrm{X}_{\mathrm{CO}}$ conversion factor.

\begin{tabular}{lcc}
\hline \hline $\begin{array}{l}M\left(\mathrm{H}_{2}\right) \\
\left(\log M_{\odot}\right)\end{array}$ & $\begin{array}{c}\mathrm{H}_{2} \mathrm{MF}_{c} \\
\left(\# \mathrm{gal} \mathrm{Mpc}^{3} \mathrm{dex}^{-1}\right)\end{array}$ & $\begin{array}{c}\mathrm{H}_{2} \mathrm{MF}_{v} \\
\left(\# \mathrm{gal} \mathrm{Mpc}^{3} \mathrm{dex}^{-1}\right)\end{array}$ \\
\hline 6.5 & -2.19 & -2.36 \\
6.75 & -2.14 & -2.14 \\
7.0 & -2.08 & -2.02 \\
7.25 & -2.01 & -1.96 \\
7.5 & -1.92 & -1.93 \\
7.75 & -1.85 & -1.94 \\
8.0 & -1.85 & -1.98 \\
8.25 & -1.96 & -2.04 \\
8.5 & -2.17 & -2.12 \\
8.75 & -2.42 & -2.24 \\
9.0 & -2.68 & -2.40 \\
9.25 & -2.94 & -2.64 \\
9.5 & -3.20 & -3.78 \\
9.75 & -3.46 & -5.2 \\
10.0 & -3.62 & -6.00 \\
\hline
\end{tabular}

The disagreement at large molecular masses is only indicative because of the lack of statistical significance in the number of objects in the HRS sample in this mass range.

It is interesting to understand which insights can be derived by comparing the results of this work with the theoretical predictions. The models by Lagos et al. (2015) are based on hydrodynamical simulations using two different prescriptions (GK11 and K13, respectively; see Lagos et al. 2015) to convert neutral to molecular Hydrogen. These prescriptions are functions of the metallicity (and therefore of the dust-to-gas ratio) and of the radiation field assumed for the cold neutral medium.

In the GK11 case, not all the resulting $\mathrm{H}_{2}$ gas is locked up in star-forming regions and 20-40\% may be present in the form of diffuse gas that is not associated with star-forming regions; a fraction of which, however, does not strongly depend on the stellar mass. In the K13 case, the fraction of molecular gas locked up in star-forming region is lower (up to 5-20\%) and it depends on the stellar mass for low values of the stellar mass. The theoretical predictions resulting from the two recipes are both compatible with the observations and the different physical processes implied by the models may be at work in the ISM of the galaxies. Both models are shown in Fig. 2.

Popping et al. (2014) adopted a semi-analytical model with two different approaches for calculating the molecular fraction of the cold neutral gas in a galaxy. The first is based on an empirical recipe dependent on the gas pressure, while the second is the same GK11 model used by Lagos et al. (2015). The number of galaxies at a low and large $\mathrm{H}_{2}$ gas mass lies far above the observed number densities. While the observational uncertainties at large masses are quite significant and larger complete samples containing data of massive galaxies need to be built before any conclusion can be drawn, the disagreement at low masses is large. To our understanding, this has more to do with an overestimation of the number of galaxies of any mass predicted by their model than to the kind of prescription used to convert the gas into a molecular mass.

We have also compared our results with those derived by Fletcher et al. (2020) using the COLD GASS survey (Saintonge et al. 2011, 2017). Strictly speaking, this comparison is only indicative because, as previously mentioned, the selection of the COLD GASS sample is not unbiased and there 


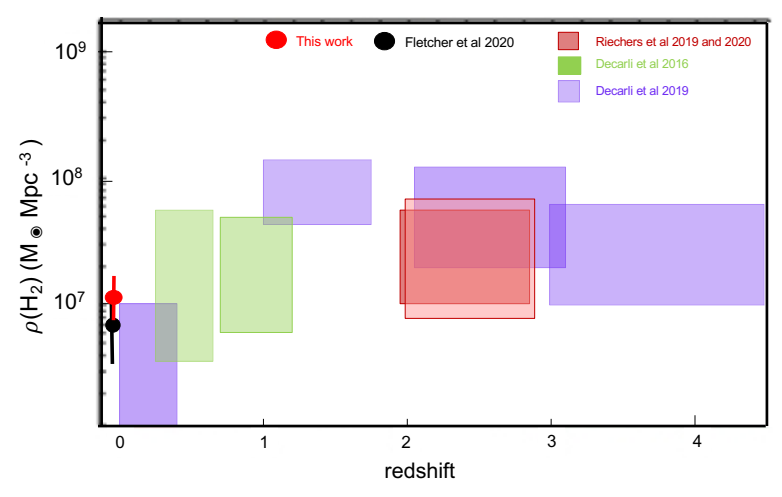

Fig. 3. Evolution of the molecular gas density as a function of redshift. Data are taken from Decarli et al. (2016, 2019), Riechers et al. (2019, 2020), and Fletcher et al. (2020). The value from this work is shown in red.

might be caveats that are not easy to understand. Our estimation lies a factor of 5 above that of Fletcher et al. (2020) at low masses $\left(\log \left(M\left(\mathrm{H}_{2}\right)\right)<8.2\right)$ and lower by the same factor at $9.5<\log \left(M\left(\mathrm{H}_{2}\right)\right)<10$.

Finally we provide an estimate of the molecular mass density in the local Universe by integrating the functions shown in Fig. 2. We find a value of $(1.6 \pm 0.6) 10^{7} M_{\odot} \mathrm{Mpc}^{-3}$ and $(1.5 \pm 0.5) 10^{7} M_{\odot} \mathrm{Mpc}^{-3}$ for our two estimated MFs with a constant and a luminosity-dependent $\mathrm{X}_{\mathrm{CO}}$, respectively. The values derived from the HRS at $z=0$ for both $M\left(\mathrm{H}_{2}\right) c$ and $M\left(\mathrm{H}_{2}\right) v$ agree within the errorbars and differ by a factor of two with that of Fletcher et al. (2020), despite the fact that the authors used a $\mathrm{X}_{\mathrm{CO}}$ factor of $\alpha_{\mathrm{CO}}=4.36 M_{\odot} /\left(\mathrm{K} \mathrm{km} \mathrm{s}^{-1} \mathrm{pc}^{2}\right.$, a value 1.2 larger than that used in this Letter $\left(\alpha_{\mathrm{CO}}=3.6\right)$ (Boselli et al. 2014b).

This result is placed in Fig. 3 where we have reported the values inferred from higher redshift surveys carried out with a blind search in ALMA data cubes (Decarli et al. 2016, 2019; Riechers et al. 2019, 2020). Strictly speaking, this graph only allows for a qualitative comparison. The conversion to molecular gas mass from the $\mathrm{CO}$ line luminosity is highly dependent on various factors of which the dominant factor is the metallicity (Bolatto et al. 2013), but even in the local Universe the environment also plays a role (for instance, see Cicone et al. 2018). The status of the interstellar medium and therefore the properties of the CO-traced molecular gas at larger redshift is highly uncertain and the assumptions made to infer a molecular gas mass for the entire galaxy do not stand on solid ground (see i.e. Papadopoulos et al. 2018). In addition to the $\mathrm{X}_{\mathrm{CO}}$ conversion factor, $\alpha_{\mathrm{CO}}=3.6 M_{\odot} /\left(\mathrm{K} \mathrm{km} \mathrm{s}^{-1} \mathrm{pc}^{2}\right)$ used in both ALMA surveys (Decarli et al. 2016, 2019; Riechers et al. 2019, 2020), to infer the $\mathrm{CO}(1-0)$ line luminosity, Decarli et al. (2019) and Riechers et al. (2019) needed to convert the observed values, $\mathrm{CO}(2-1), \mathrm{CO}(3-2)$, and $\mathrm{CO}(4-3)$, into $\mathrm{CO}(1-0)$ by making an assumption on the CO SLED. The used template is that of a standard CO SLED of star forming main sequence galaxies.

It is important to note that this latter argument is no longer valid for the values reported in Fig. 3 in the redshift range of $z=2-3$ by Riechers et al. (2020) who measured the CO(1$0)$ line emission with the VLA. An additional source of uncertainties of difficult quantification is the cosmic variance, which is associated with the small sky areas samples by the ALMA surveys.

This means that the values of the molecular mass densities at various redshift is highly uncertain. Within the (large) errorbars, the inferred values of the molecular mass density in the local
Universe are not different from the values derived in surveys targeting higher redshift galaxies. There is no evidence at present of a large evolution of the molecular gas from $z=0$ up to $z \sim 4$.

Acknowledgements. This research has made use of data from the HRS project. HRS is a Herschel Key Programme utilising guaranteed time from the SPIRE instrument team, ESAC scientists and a mission scientist. The HRS data was accessed through the Herschel Database in Marseille (HeDaM http://hedam.lam.fr) operated by CeSAM and hosted by the Laboratoire d'Astrophysique de Marseille. P.A. warmly thanks the NRO staff at the Nobeyama Observatory for their fundamental help in performing the observations. She warmly thanks NAOJ-Chile for her stay at Mitaka where this work has been started. This research benefited from discussions happened at the MIAPP workshop "Galaxy Evolution in a New Era of HI Surveys" held in 2019 and supported by the Munich Institute for Astro- and Particle Physics (MIAPP) which is funded by the Deutsche Forschungsgemeinschaft (DFG, German Research Foundation) under Germany Excellence Strategy - EXC-2094 - 390783311. This work makes use of SW routines contained in the $R$-package, $R$ is available as Free Software under the terms of the Free Software Foundation's GNU General Public License in source code form, see https://www.r-project.org/ about.html. This publication makes use of data products from the Two Micron All Sky Survey, which is a joint project of the University of Massachusetts and the Infrared Processing and Analysis Center/California Institute of Technology, funded by the National Aeronautics and Space Administration and the National Science Foundation.

\section{References}

Andreani, P., Spinoglio, L., Boselli, A., Ciesla, L., \& Vio, R. 2014, A\&A, 566, 70

Andreani, P., Boselli, A., Ciesla, L., Vio, R., et al. 2018, A\&A, 617, 33

Bigiel, F., Leroy, A., Walter, F., et al. 2008, AJ, 136, 2846

Bolatto, A. D., Wolfire, M., \& Leroy, A. K. 2013, ARA\&A, 51, 207

Boselli, A., Eales, S., Cortese, L., et al. 2010, PASP, 122, 261

Boselli, A., Ciesla, L., Cortese, L., et al. 2012, A\&A, 540, A54

Boselli, A., Cortese, L., \& Boquien, M. 2014a, A\&A, 564, A65

Boselli, A., Cortese, L., Boquien, M., et al. 2014b, A\&A, 564, A66

Boselli, A., Cortese, L., Boquien, M., et al. 2014c, A\&A, 564, A67

Boselli, A., Fossati, M., Gavazzi, G., et al. 2015, A\&A, 579, 102

Cicone, C., Severgnini, P., Papadopoulos, P. P., et al. 2018, ApJ, 863, 143

Ciesla, L., Boquien, M., Boselli, A., et al. 2014, A\&A, 565, A128

Cortese, L., Ciesla, L., Boselli, A., et al. 2012, A\&A, 540, A52

Dave, R., Rafieferantsoa, M. H., Thompson, R. J., et al. 2017, MNRAS, 467, 115

Decarli, R., Walter, F., Aravena, M., et al. 2016, ApJ, 833, A69

Decarli, R., Walter, F., Gónzalez-López, J., et al. 2019, ApJ, 882, 138

Dessauges, M., Zamojski, M., Rujopakarn, W., et al. 2017, A\&A, 605, A81

Fletcher, T., Saintonge, A., Soares, P. S., \& Pontzen, A. 2020, MNRAS, in press [arXiv:2002.04959]

Hofert, M., Kojadinovic, I., Mächler, M., \& Yan, J. 2018, Elements of Copula Modeling with R (New York: Springer)

Johnston, R. 2011, A\&ARv, 19, 41

Kennicutt, R. C., \& Evans, N. J. 2012, ARA\&A, 50, 531

Keres, D., Yun, M. S., \& Young, J. S. 2003, ApJ, 582, 659

Lagos, C. del P., Crain, R. A., Schaye, J., et al. 2015, MNRAS, 452, 3815

Lagos, C. del P., Theuns, T., Schaye, J., et al. 2016, MNRAS, 459, 2632

Minamidani, T., Nishimura, A., Miyamoto, Y., et al. 2016, Proc. SPIE, 9914, $99141 Z$

Mo, H. J., Mao, S., \& White, S. D. M. 1998, MNRAS, 295, 319

Mo, H. J., van den Bosch, F. K., \& White, S. D. M. 2010, Galaxy Formation and Evolution (Cambridge: Cambridge University Press)

Nakajima, T., Kimura, K., Nishimura, A., et al. 2013, PASP, 125, 252

Obreschkow, D., \& Rawlings, S. 2009, MNRAS, 394, 1857

Papadopoulos, P. P., Bisbas, T., \& Zhang, Z. Y. 2018, MNRAS, 478, 1716

Popping, G., Somerville, R. S., \& Trager, S. 2014, MNRAS, 442, 2398

Riechers, D. A., Pavesi, R., Sharon, C. E., et al. 2019, ApJ, 872, 7

Riechers, D. A., Boogaard, L. A., Decarli, R., et al. 2020, ApJ, 896, L21

Saintonge, A., Kauffmann, G., Kramer, C., et al. 2011, MNRAS, 415, 32

Saintonge, A., Catinella, B., Tacconi, L. J., et al. 2017, ApJS, 233, 22

Schlegel, D. J., Finkbeiner, D. P., \& Davis, M. 1998, ApJS, 500, 525

Schruba, A., Leroy, A. K., Walter, F., et al. 2011, AJ, 142, 37

Skrutskie, M. F., Cutri, R. M., Stiening, R., et al. 2006, AJ, 131, 1163

Solomon, P. M., \& van den Bout, P. A. 2005, ARA\&A, 43, 677

Somerville, R., \& Dave, R. 2015, ARA\&A, 53, 51

Sorai, K., Kuno, N., Muraoka, K., et al. 2019, PASJ, 71, S14

Vio, R., Fasano, G., Lazzarin, M., \& Lessi, O. 1994, A\&A, 289, 640 